\title{
Hausdorff Convergence and Asymptotic Estimates of the Spectrum of a Perturbed Operator
}

\author{
T. A. Mel'nyk
}

\begin{abstract}
A family of self-adjoint compact operators $A_{\varepsilon}(\varepsilon>0)$ acting in Hilbert spaces $\mathcal{H}_{\varepsilon}$ is considered. The asymptotic behaviour as $\varepsilon \rightarrow 0$ of eigenvalues and eigenvectors of the operators $A_{\varepsilon}$ is studied; the limiting operator $A_{0}: \mathcal{H}_{0} \mapsto \mathcal{H}_{0}$ is non-compact. Asymptotic estimates of the differences between eigenvalues of $A_{\varepsilon}$ and points of the spectrum $\sigma\left(A_{0}\right)$ (both of the discrete spectrum and the essential one) are obtained. Asymptotic estimates for eigenvectors of $A_{\varepsilon}$ are also proved.
\end{abstract}

Keywords: Spectrum, asymptotic estimates, perturbed operators

AMS subject classification: Primary 47A55, secondary 47A75, 35B25

\section{Introduction}

The task of eigenvalue perturbation theory is to study the asymptotic behaviour of eigenvalues and eigenfunctions of some perturbed operator $A(\varepsilon)$ as $\varepsilon \rightarrow 0$, where $\varepsilon$ is usually a positive parameter of the perturbation. If the domains of definition of $A(\varepsilon)$ and the limiting operator $A(0)$ are the same, i.e. $D(A(\varepsilon))=D(A(0))$ for any $\varepsilon>0$, then this behaviour depends essentially on the convergence $A(\varepsilon)$ to the limiting operator $A(0)$. For many problems $A(\varepsilon)$ must converge strongly to $A(0)$.

In the papers $[46,50]$, nice reviews of eigenvalue perturbation theory were given, especially, for problems connected to quantum mechanics, where we usually have different perturbations of potentials. There were discussed also some problems, which do not fit into the scheme of Kato's perturbation theory. The main reason, why this theory cannot be applied, is the essential spectrum of the limiting operator.

In the classic book by Kato [17] there are two examples (Examples 1.19 and 1.20 in Section VII) with the same kind of perturbation (small parameter near the higher derivative), but the asymptotic behaviour of the spectrum is completely different. These examples show that the spectrum of a perturbed operator can behave very complex itself at singular perturbation. In [47] an abstract operator scheme of such kind of perturbation was proposed. The main assumption is the uniformly convergence of the resolvents. Spectral problems of homogenization theory, problems with perturbed boundary conditions, spectral stiff problems fit also into this scheme. A new aspect (high frequency

T. A. Mel'nyk: Kyiv Univ., Fac. Math. \& Mech., Volodymyrska str. 64, 01033 Kyiv, Ukraine melnyk@imath.kiev.ua 
convergence) of the asymptotic behaviour of the spectrum of stiff problems was studied in $[4,23]$. Using the WKB-method, asymptotic expansions for eigenvalues and eigenfunctions were constructed in [23].

A lot of cases of asymptotic behaviour of the spectrum appear in spectral problems with concentrated masses [12, 13, 20 - 22, 40, 48]. The asymptotic behaviour depends on the dimension of the space and of the region, where we have the density perturbation.

Domain perturbation. It is an interesting problem to study the behaviour of the eigenvalues and eigenfunctions of certain differential operators when the domain is perturbed. If this perturbation is smooth and in some sense small, then with the help of a family of diffeomorphisms we can reduce the problem to the study of the behaviour of the spectrum of a family of operators defined in the same domain; and the coefficients of these operators behave well enough. On the other hand, there are many problems, in which the domain perturbation is not smooth and small, and it is not possible to use this method. In [41], for a simple eigenvalue of the Neumann problem in $\Omega_{\varepsilon}$, the difference between the asymptotic formulas in the case of smooth and non-smooth (step-like) perturbations was shown.

Moreover, the asymptotic behaviour of the spectrum depends essentially on the kind of boundary conditions that we impose. For eigenvalues to depend continuously on a variation of the domain, Dirichlet conditions allow much more general perturbations of the domain than Neumann conditions (see, for example, $[2,3,9,14,15]$ ).

The asymptotic behaviour of eigenvalues of boundary value problems in domains with a small cavity of order $\varepsilon$, i.e. $\Omega_{\varepsilon}=\Omega \backslash \omega_{\varepsilon}$, was investigated in $[25,43]$ (for the Laplace operator) and in [16] (for systems of differential operators of high order).

There are many publications which have been aimed at spectral problems in $\varepsilon$ periodically perforated domains with different boundary conditions on the holes (see [29, $42,51,52]$ and references there). In [29] complete asymptotic expansions with respect to a small parameter $\varepsilon$ were constructed for eigenvalues and eigenfunctions of spectral boundary value problems of Steklov, Neumann, and Dirichlet types. In [42] an abstract operator scheme for the investigation of the behaviour of eigenvalues and eigenfunctions of operators acting in different spaces was proposed. Using this scheme, it is possible to justify the asymptotic behaviour and to obtain asymptotic estimates for eigenvalues and eigenfunctions of boundary value problems with various kinds of perturbations (see [42: Section III]). Here we cite some new papers, in which this scheme was also applied: spectral problems with quickly oscillating boundary conditions [8], spectral problems in domains perforated along the boundary [5], and spectral problems in strongly nonuniform thin perforated domains with rapidly varying thickness [26].

There are many papers that deal with the asymptotic investigation of spectral boundary value problems in junctions consisting of a finite number of domains with different limit dimensions $[2,7,11,14,15,18,19,39]$. Asymptotics for low eigenvalues were found in $[11,18,19]$. In $[2,14,15]$ the behaviour of eigenvalues of Neumann Laplacians was studied in perturbed domains $\Omega_{\varepsilon}$, for which $\Omega \subset \Omega_{\varepsilon}$ and the Lebesgue measure of $\Omega_{\varepsilon} \backslash \Omega$ tends to 0 as $\varepsilon \rightarrow 0$. There it was shown that besides of eigenvalues of the Neumann Laplacian in $\Omega$ there are other accumulation points. More full results were obtain in [7], where it was shown that the solutions of three-dimensional elastic structure problems converge to solutions of a "coupled" eigenvalue problem of a new 
type. A similar limiting problem was obtained in [31] for the Laplacian, but the junction body is degenerated as $\varepsilon \rightarrow 0$.

Spectral boundary value problems in junctions, whose number of components increases as $\varepsilon \rightarrow 0$, have own specific difficulties. The main reason is the special character of the connectedness of such thick junctions, which are the union of some domain and a large number of $\varepsilon$-periodically situated thin domains: there are points in a thick junction, which are at a short distance of order $O(\varepsilon)$, but the length of all curves, which connect these points in the junction, is of order $O(1)$. Because of this, many new effects appear in the asymptotic behavior of solutions of boundary value problems in thick junctions. As was shown in $[6,10,49]$, the limiting operators are not compact for spectral boundary value problems in thick junctions, and these operators can have both points of the discrete spectrum and points of the essential spectrum. As a consequence, "...an exhaustive study of the spectral properties will not be done" (see [10: p. 163]). In $[27,28,30-32,34-37]$ the full structure of the spectrum of limiting operators was found, and a new method was proposed for the investigation of the asymptotic behavior of eigenvalues and eigenfunctions of boundary value problems in thick junctions of different types. There were obtained asymptotic estimates both for eigenvalues and corresponding eigenfunctions. For these problems there are both common singularities in the asymptotic investigation and own particular singularities for each problem that are defined by the type of a thick junction (for more detail reviews, see [35, 36]). In [36] asymptotic estimates were obtained for solutions of a spectral problem in thick junction of type $3: 1: 1$, when there are simultaneously three kind of perturbations: stiff perturbation, density perturbation, and domain perturbation.

Here we present an abstract scheme of investigation of the asymptotic behaviour of eigenvalues and eigenvectors of some family of abstract operators $\left\{A_{\varepsilon}\right\}$ acting in different spaces. This scheme generalizes the procedure of justification of the asymptotic behaviour of eigenvalues and eigenvectors of boundary value problems in thick junctions of different types [27, 28, $30-32,34-37]$. Using this scheme, we can obtain asymptotic estimates both for eigenvalues and for eigenfunctions. Of course, the scheme can be applied to other perturbed spectral problems, which satisfy conditions represented below.

Cases, in which the essential spectrum is changed by perturbation into the discrete spectrum, appeared in many other perturbed problems (see [46], [17: Section VII/Example 1.19], [4, 13, 14, 23], [24: Section III.4], and [1, 45]). Similar effects, namely, spectral pollution could appear in finite element approximation of eigenvalues and eigenfunctions of thin elastic shell problems in membrane approximation (see [44] and references therein). The richness of such problems and different methods of investigation is a consequence of the various kinds of perturbations. Most part of these studies was made under the restrictions that the strong convergences of the corresponding spectral families and domains of definition both for the limiting operator and for the perturbed operators are the same. In [1] the authors reduced some perturbed problem in a perforated domain to the asymptotic investigation of a sequence of such operators $\left\{T^{\varepsilon, K}: K \in \mathbb{N}\right\}$ with fixed domain and co-domain, each of them converging strongly to an operator $\widetilde{T}^{K}$ as $\varepsilon \rightarrow 0$. In [49: Section V.11 - V.13], the authors preferred to study corresponding spectral families and to propose a new method; the idea is based on 
the convergence of solutions of evolution problems in time and on Fourier and Laplace transforms of the corresponding solutions. This method was also used in $[23,44,46]$.

Our scheme with respect to his ideology is close to the scheme in [42: Section III.1]. There exist four principal distinctions between these schemes, which are discussed in Subsection 1.2.

\section{Statement of the problem}

1.1 The family of self-adjoint compact operator $\left\{A_{\varepsilon}: \varepsilon>0\right\}$. Eigenvalue perturbation theory originated with the description of proper oscillations of mechanical systems. Usually oscillating processes in domains depending on a small parameter $\varepsilon$ are described by the problem

$$
\left.\begin{array}{c}
\left(\frac{d^{2} U_{\varepsilon}}{d t^{2}}, v\right)_{\mathcal{V}_{\varepsilon}}+a_{\varepsilon}\left(U_{\varepsilon}, v\right)=0 \quad\left(v \in \mathcal{H}_{\varepsilon}\right) \\
U_{\varepsilon}(0)=\phi_{1}, \frac{d U_{\varepsilon}}{d t}(0)=\phi_{2}
\end{array}\right\}
$$

where $a_{\varepsilon}$ is a bilinear, symmetric, continuous, and coercive form on a separable Hilbert space $\mathcal{H}_{\varepsilon}$, which is densely and compactly embedded into a Hilbert space $\mathcal{V}_{\varepsilon}$, and where the constants $C_{1}, C_{2}, C_{3}$ in the inequalities

$$
\left.\begin{array}{rl}
\left|a_{\varepsilon}(u, v)\right| & \leq C_{1}\|u\|_{\mathcal{H}_{\varepsilon}}\|v\|_{\mathcal{H}_{\varepsilon}} \\
a_{\varepsilon}(u, u) & \geq C_{2}\|u\|_{\mathcal{H}_{\varepsilon}}^{2} \\
\|u\|_{\mathcal{V}_{\varepsilon}} & \leq C_{3}\|u\|_{\mathcal{H}_{\varepsilon}}
\end{array}\right\} \quad\left(u, v \in \mathcal{H}_{\varepsilon}\right)
$$

are independent of the small parameter $\varepsilon>0$.

Remark 1. It should be noted that here and further all constants $c_{i}$ and $C_{i}$ in asymptotic inequalities are independent of the parameter $\varepsilon$.

Obviously, the bilinear form $a_{\varepsilon}$ defines a new scalar product in $\mathcal{H}_{\varepsilon}$; the corresponding norm is equivalent to the norm in $\mathcal{H}_{\varepsilon}$, and in this case the constants are also independent of the small parameter $\varepsilon$.

Let $(u, v)_{\mathcal{H}_{\varepsilon}}=a_{\varepsilon}(u, v)$. Then the corresponding proper oscillation problem (we look for oscillations of the form $\left.U_{\varepsilon}=\exp (-i \sqrt{\lambda(\varepsilon)} t) u^{\varepsilon}\right)$ is reduced to the spectral problem

$$
\lambda(\varepsilon) A_{\varepsilon}\left(u^{\varepsilon}\right)=u^{\varepsilon} \quad \text { in } \mathcal{H}_{\varepsilon}
$$

where $A_{\varepsilon}: \mathcal{H}_{\varepsilon} \mapsto \mathcal{H}_{\varepsilon}$ is a self-adjoint, positive, compact operator, which is defined by the formula

$$
\left(A_{\varepsilon} u, v\right)_{\mathcal{H}_{\varepsilon}}=(u, v)_{\mathcal{V}_{\varepsilon}} \quad\left(u, v \in \mathcal{H}_{\varepsilon}\right)
$$

It is easy to see that $\sup _{\varepsilon>0}\left\|A_{\varepsilon}\right\| \leq C_{3}^{2}$.

So, for each fixed $\varepsilon>0$, all characteristic values of the operator $A_{\varepsilon}$ make up the sequence

$$
0<\frac{1}{C_{3}^{2}} \leq \lambda_{1}(\varepsilon) \leq \ldots \leq \lambda_{n}(\varepsilon) \leq \ldots \rightarrow+\infty \quad(n \rightarrow \infty)
$$


with the classical convention of repeated values. Let $\left\{u_{n}^{\varepsilon}: n \in \mathbb{N}\right\}$ be the set of corresponding eigenvectors, which are orthonormal in $\mathcal{V}_{\varepsilon}$, i.e.

$$
\left(u_{n}^{\varepsilon}, v_{m}^{\varepsilon}\right) \mathcal{V}_{\varepsilon}=\delta_{n, m} \quad(n, m \in \mathbb{N})
$$

where $\delta_{n, m}$ is the Kronecker symbol.

The aim of the present paper is to study the asymptotic behavior of the characteristic values $\lambda_{n}(\varepsilon)$ and the eigenvectors $u_{n}^{\varepsilon}$ as $\varepsilon \rightarrow 0$, and to find other limiting points of the spectrum of the operator $A_{\varepsilon}$ under conditions imposed below.

1.1 Limiting operator. Let $\mathcal{H}_{0}$ be a separable Hilbert space, which is densely and only continuously embedded into a Hilbert space $\mathcal{V}_{0}$. Since this embedding is not compact, the operator $A_{0}: \mathcal{H}_{0} \mapsto \mathcal{H}_{0}$ defined by

$$
\left(A_{0} u, v\right)_{\mathcal{H}_{0}}=(u, v)_{\mathcal{V}_{0}} \quad\left(u, v \in \mathcal{H}_{0}\right)
$$

is self-adjoint, positive, and continuous only. As was shown in $[6,27,28,30-32,34$, 36], the spectrum $\sigma\left(A_{0}\right)$ of the limiting operator consists of the discrete part $\sigma_{d}\left(A_{0}\right)$ formed by finite-to-one isolated eigenvalues $\frac{1}{\mu}$, and of the essential spectrum $\sigma_{\text {ess }}\left(A_{0}\right)=$ $\sigma\left(A_{0}\right) \backslash \sigma_{d}\left(A_{0}\right)$. Two cases of their reciprocal situation are possible:

- The essential spectrum consists of accumulation points, which divide the eigenvalues into non-increasing sequences.

- There is a finite number of intervals on the positive semi-axis, where are situated non-increasing sequences of eigenvalues with finite multiplicity, and the essential spectrum is located between these intervals.

We consider the second case, which is more general, and assume in addition that there are only two such intervals. Then the eigenvalues of the operator $A_{0}$ form two sequences

$$
\begin{gathered}
\frac{1}{b_{1}} \leftarrow \ldots \leq \frac{1}{\mu_{n}^{(1)}} \leq \ldots \frac{1}{\mu_{1}^{(1)}} \\
0<\frac{1}{b_{3}} \leftarrow \ldots \leq \frac{1}{\mu_{n}^{(2)}} \leq \ldots \frac{1}{\mu_{1}^{(2)}}<\frac{1}{b_{2}}<\frac{1}{b_{1}}
\end{gathered}
$$

as $n \rightarrow \infty$, and the essential spectrum $\sigma_{e s s}\left(A_{0}\right)$ is equal to the union $\left[0, b_{3}^{-1}\right] \cup\left[b_{2}^{-1}, b_{1}^{-1}\right]$.

Let $v_{n}^{(k)} \quad(n \in \mathbb{N} ; k=1,2)$ be the corresponding eigenvectors, which are assumed to be in a Hilbert space $\mathcal{Z}_{0}$ which is densely and continuously embedded into $\mathcal{H}_{0}$ and compactly embedded into $\mathcal{V}_{0}$. Let these eigenvectors be orthonormalized in $\mathcal{V}_{0}$, i.e.

$$
\left(v_{n}^{(k)}, v_{m}^{(k)}\right) \mathcal{V}_{0}=\delta_{n, m} \quad \text { and } \quad\left(v_{n}^{(1)}, v_{m}^{(2)}\right) \mathcal{V}_{0}=0 \quad(n, m \in \mathbb{N} ; k=1,2) .
$$

1.2 Connection conditions between the spaces. First let us introduce some notation. By $N\left(\frac{1}{\mu}, A_{0}\right)$ denote the proper subspace corresponding to the eigenvalue $\frac{1}{\mu}$ of the operator $A_{0}$. By $\left(u^{\varepsilon}, \lambda(\varepsilon), \Lambda\right) \quad(\varepsilon>0)$ denote a sequence, whose components are 
respectively the normed eigenvector $u^{\varepsilon}$, the corresponding characteristic number of the operator $A_{\varepsilon}$, and the limit number $\Lambda=\lim _{\varepsilon \rightarrow 0} \lambda(\varepsilon)$. In view of $(3), \Lambda \neq 0$.

Assume that the following conditions (C1) - (C6) are satisfied.

(C1) There exists a linear continuous operator $\mathbf{S}_{\varepsilon}: \mathcal{V}_{0} \mapsto \mathcal{V}_{\varepsilon}$ with $\mathbf{S}_{\varepsilon}\left(\mathcal{Z}_{0}\right) \subset \mathcal{H}_{\varepsilon}$ such that

$$
\left.\begin{array}{rl}
\left\|\mathbf{S}_{\varepsilon} u\right\|_{\mathcal{V}_{\varepsilon}} \leq c_{1}\|u\|_{\mathcal{V}_{0}} & \left(u \in \mathcal{V}_{0}\right) \\
\left\|\mathbf{S}_{\varepsilon} u\right\|_{\mathcal{H}_{\varepsilon}} \leq c_{1}\|u\|_{\mathcal{Z}_{0}} & \left(u \in \mathcal{Z}_{0}\right)
\end{array}\right\}
$$

where the constant $c_{1}>0$ depends neither on $\varepsilon$ nor on $u$.

(C2) There exists a linear continuous operator $\mathbf{P}_{\varepsilon}: \mathcal{H}_{\varepsilon} \mapsto \mathcal{Z}_{0}$ such that, for any sequence $\left\{\left(u^{\varepsilon}, \lambda(\varepsilon), \Lambda\right): \varepsilon>0\right\}$ with $\frac{1}{\Lambda} \notin \sigma_{\text {ess }}\left(A_{0}\right)$, there exist some constants $c_{2}>0$ and $\varepsilon_{0}>0$ such that

$$
\left\|\mathbf{P}_{\varepsilon} u^{\varepsilon}\right\|_{\mathcal{Z}_{0}} \leq c_{2}(\Lambda)\left\|u^{\varepsilon}\right\|_{\mathcal{H}_{\varepsilon}}
$$

for all $\varepsilon \in\left(0, \varepsilon_{0}\right)$. If $v \in \mathcal{Z}_{0}$, then

$$
\left\|\mathbf{P}_{\varepsilon}\left(\mathbf{S}_{\varepsilon} v\right)\right\|_{\mathcal{Z}_{0}} \leq c_{3}\left\|\mathbf{S}_{\varepsilon} v\right\|_{\mathcal{H}_{\varepsilon}}
$$

for all $\varepsilon \in\left(0, \varepsilon_{0}\right)$ where the constant $c_{3}>0$ depends neither on $\varepsilon$ nor on $v$ and $\mathbf{P}_{\varepsilon}\left(\mathbf{S}_{\varepsilon} v\right) \rightarrow$ $v$ in $\mathcal{V}_{0}$ as $\varepsilon \rightarrow 0$.

(C3) For any sequence $\left\{\left(u^{\varepsilon}, \lambda(\varepsilon), \Lambda\right): \varepsilon>0\right\}$ with $\frac{1}{\Lambda} \notin \sigma_{\text {ess }}\left(A_{0}\right)$ and for any subsequence $\left\{\varepsilon^{\prime}\right\}$ such that $\mathbf{P}_{\varepsilon^{\prime}} u^{\varepsilon^{\prime}} \rightarrow u^{0}$ weakly in $\mathcal{Z}_{0}$,

$$
\lim _{\varepsilon^{\prime} \rightarrow 0}\left(u^{\varepsilon^{\prime}}, \mathbf{S}_{\varepsilon^{\prime}} v\right)_{\mathcal{H}_{\varepsilon^{\prime}}}=\left(u^{0}, v\right)_{\mathcal{H}_{0}}
$$

for all $v \in \mathcal{Z}_{0}$.

(C4) If, for some vectors $w^{\varepsilon}, v^{\varepsilon} \in \mathcal{H}_{\varepsilon}, \mathbf{P}_{\varepsilon} w^{\varepsilon} \rightarrow w^{0}$ in $\mathcal{V}_{0}$ and $\mathbf{P}_{\varepsilon} v^{\varepsilon} \rightarrow v^{0}$ in $\mathcal{V}_{0}$ as $\varepsilon \rightarrow 0$, then $\lim _{\varepsilon \rightarrow 0}\left(w^{\varepsilon}, v^{\varepsilon}\right) \mathcal{V}_{\varepsilon}=\left(w^{0}, v^{0}\right) \mathcal{V}_{0}$.

(C5) There is a number $\delta_{0}>0$ such that for any $\frac{1}{\mu} \in \sigma_{d}\left(A_{0}\right)$ there exists a linear operator $R_{\varepsilon}: N\left(\frac{1}{\mu}, A_{0}\right) \mapsto \mathcal{H}_{\varepsilon}$ such that, for all $v \in N\left(\frac{1}{\mu}, A_{0}\right)$ with $\|v\|_{\mathcal{V}_{0}}=1$,

$$
R_{\varepsilon} v=\mathbf{S}_{\varepsilon} v+\mathcal{O}(\varepsilon) \text { in } \mathcal{V}_{\varepsilon} \quad \text { and } \quad\left\|R_{\varepsilon} v\right\|_{\mathcal{H}_{\varepsilon}}=c_{v}+\mathcal{O}(\varepsilon) \text {, }
$$

and there exists some constant $c_{3}>0$ such that

$$
\left\|\mu A_{\varepsilon}\left(R_{\varepsilon} v\right)-R_{\varepsilon} v\right\|_{\mathcal{H}_{\varepsilon}} \leq c_{3} \varepsilon^{\delta_{0}}
$$

for any $\varepsilon \in\left(0, \varepsilon_{0}\right)$.

(C6) There exist $\delta_{1}>0$ such that for any $\frac{1}{\mu} \in \sigma_{\text {ess }}\left(A_{0}\right)$ there exists constants $c_{4}>0$ and $\varepsilon_{0}>0$ and an element $w_{\varepsilon} \in \mathcal{H}_{\varepsilon}$ with $\left\|w_{\varepsilon}\right\|_{\mathcal{H}_{\varepsilon}}=1+\mathcal{O}(\varepsilon)$ such that

$$
\left\|\mu A_{\varepsilon} w_{\varepsilon}-w_{\varepsilon}\right\|_{\mathcal{H}_{\varepsilon}} \leq c_{4} \varepsilon^{\delta_{1}}
$$

for all $\varepsilon \in\left(0, \varepsilon_{0}\right)$. 
For better understanding, we write the diagram

in which the imbedding $\mathcal{H} \subset \mathcal{V}$ means that the space $\mathcal{H}$ is densely and only continuously embedded into $\mathcal{V}$, but the imbedding $\mathcal{H} \subset \subset \mathcal{V}$ is compact in addition. It should be stressed here that the operator $\mathbf{P}_{\varepsilon}$ is not uniformly bounded with respect to $\varepsilon$; it is uniformly bounded only on some sequences (see condition (C2)). We note also that $\mathcal{Z}_{0} \subset \subset \mathcal{V}_{0}$, and usually $\delta_{1} \leq \delta_{0} \leq 1$.

Remark 2. In this remark we comment the main distinctions between the scheme in [42: Subsection III.1]:

(i) The limiting operator $A_{0}$ in [42] is compact.

(ii) The family of operator $\left\{A_{\varepsilon}\right\}$ is uniformly compact. The facts of this condition for spectral problems in domains depending on a small parameter $\varepsilon$ mean that there exists an extension operator in a domain, which is independent of $\varepsilon$, and this operator is bounded uniformly with respect to $\varepsilon$ in the corresponding Sobolev norm. The uniformly boundedness of extension operators is a necessary condition in statements of some problems (see [54]). For other problems, such extension operators exist, for example, for domains that are $\varepsilon$-periodically perforated by holes with the diameter of order $\varepsilon[1,5,26,42,51,52]$. But for thick junctions, there exist no extension operators that are bounded uniformly in $\varepsilon$ (see, for instance, $[32,35,36]$ ). This is one of the main difficulties in the investigation of boundary value problems in thick junctions.

(iii) There is no analogy in our scheme to condition (C3) in [42]. Besides, condition (C3) is not true for boundary value problems in thick junctions (see [33]).

(iv) The scheme in [42] deals only with two spaces $H_{\varepsilon}$ and $H_{0}$ where the operators $A_{\varepsilon}$ and $A_{0}$ act, respectively.

Further we will repeatedly use [53: Lemma 12], which has wide applications for the approximation of eigenvalues and eigenvectors of self-adjoint compact operators. Therefore, we recall this lemma here.

Lemma (Vishik and Lyusternik [53: Lemma 12]). Let $\mathcal{A}: H \mapsto H$ be a continuous linear compact self-adjoint operator in a Hilbert space H. Suppose there exist a number $\mu>0$ and a vector $u \in H$ such that $\|u\|_{H}=1$ and

$$
\|\mathcal{A} u-\mu u\|_{H} \leq \alpha \quad(\alpha>0) .
$$

Then there exists an eigenvalue $\mu_{n}$ of the operator $\mathcal{A}$ such that $\left|\mu_{n}-\mu\right| \leq \alpha$. Moreover, for any $d>\alpha$ there exists a vector $\bar{u}$ such that

$$
\|u-\bar{u}\|_{H} \leq 2 \alpha d^{-1}, \quad\|\bar{u}\|_{H}=1,
$$

and $\bar{u}$ is a linear combination of eigenvectors corresponding to all eigenvalues of $\mathcal{A}$ from the segment $[\mu-d, \mu+d]$. 


\section{Low frequency convergence}

Proper oscillations, which correspond to the fundamental frequencies $\sqrt{\lambda_{n}(\varepsilon)}$ at fixed indices $n$, are called low frequency oscillations, and the corresponding frequencies are called low frequencies. We introduce a new characteristic for low frequencies (see [34]).

Definition 1. The value

$$
\mathcal{T}=\sup _{n \in \mathbb{N}} \varlimsup_{\varepsilon \rightarrow 0} \lambda_{n}(\varepsilon)
$$

is called the threshold of low frequencies.

Theorem 1. For any fixed index $n \in \mathbb{N}$,

$$
\lambda_{n}(\varepsilon) \rightarrow \mu_{n}^{(1)} \quad(\varepsilon \rightarrow 0)
$$

and there exists a subsequence of the sequence $\{\varepsilon\}$ (still denoted by $\{\varepsilon\}$ ) such that

$$
\mathbf{P}_{\varepsilon} u_{n}^{\varepsilon} \rightarrow \tilde{v}_{n}^{(1)} \quad \text { weakly in } \mathcal{Z}_{0} \text { as } \varepsilon \rightarrow 0
$$

where $\tilde{v}_{n}^{(1)}$ is an eigenvector of the operator $A_{0}$ which corresponds to the characteristic value $\mu_{n}^{(1)}$ from sequence (6). Moreover, the eigenvectors $\left\{\tilde{v}_{n}^{(1)}\right\}$ are orthonormalized in $\mathcal{V}_{0}$, i.e. $\left(\tilde{v}_{n}^{(1)}, \tilde{v}_{m}^{(1)}\right) \mathcal{V}_{0}=\delta_{n, m} \quad(n, m \in \mathbb{N})$.

\section{Proof.}

1. Take any characteristic value $\mu_{n}^{(1)}$ of the operator $A_{0}$ and a corresponding eigenvector $v_{n}^{(1)}$, normalized by (8). Due to condition (C5) there exists a vector $R_{\varepsilon}^{n}:=$ $R_{\varepsilon} v_{n}^{(1)} \in \mathcal{H}_{\varepsilon}$ such that

$$
R_{\varepsilon}^{n}=\mathbf{S}_{\varepsilon} v_{n}^{(1)}+\mathcal{O}(\varepsilon) \text { in } \mathcal{V}_{\varepsilon}, \quad\left\|R_{\varepsilon}^{n}\right\|_{\mathcal{H}_{\varepsilon}}=c_{n}+\mathcal{O}(\varepsilon)
$$

and

$$
\left\|\mu_{n}^{(1)} A_{\varepsilon}\left(R_{\varepsilon}^{n}\right)-R_{\varepsilon}^{n}\right\|_{\mathcal{H}_{\varepsilon}} \leq c_{3} \varepsilon^{\delta_{0}} .
$$

Since $\mathbf{P}_{\varepsilon}\left(\mathbf{S}_{\varepsilon} v_{n}^{(1)}\right) \rightarrow v_{n}^{(1)}$ in $\mathcal{V}_{0}$ as $\varepsilon \rightarrow 0$ (see condition $(\mathrm{C} 2)$ ), it follows from condition (C4) that

$$
\lim _{\varepsilon \rightarrow 0}\left\|\mathbf{S}_{\varepsilon} v_{n}^{(1)}\right\|_{\mathcal{V}_{\varepsilon}}^{2}=\left\|v_{n}^{(1)}\right\|_{\mathcal{V}_{0}}^{2}=1
$$

Taking into account these facts and the continuity of the imbedding $\mathcal{H}_{\varepsilon} \subset \mathcal{V}_{\varepsilon}$, we get

$$
0<c_{0} \leq c\left\|\mathbf{S}_{\varepsilon} v_{n}^{(1)}\right\|_{\mathcal{V}_{\varepsilon}}^{2} \leq\left\|R_{\varepsilon}^{n}\right\|_{\mathcal{H}_{\varepsilon}}^{2} \leq c_{n}^{\prime}
$$

at $\varepsilon$ small enough, where the constant $c_{0}$ depends neither on $\varepsilon$ nor on $v_{n}^{(1)}$. Now using (10), we derive from (9)

$$
\left\|A_{\varepsilon}\left(\frac{R_{\varepsilon}^{n}}{\left\|R_{\varepsilon}^{n}\right\|_{\mathcal{H}_{\varepsilon}}}\right)-\frac{1}{\mu_{n}^{(1)}} \frac{R_{\varepsilon}^{n}}{\left\|R_{\varepsilon}^{n}\right\|_{\mathcal{H}_{\varepsilon}}}\right\|_{\mathcal{H}_{\varepsilon}} \leq c \varepsilon^{\delta_{0}} .
$$


Applying the first part of [53: Lemma 12] to (11), we conclude that every small neighborhood of each characteristic value $\mu_{n}^{(1)}$ contains a characteristic value of the operator $A_{\varepsilon}$. Since we have a denumerable set of characteristic values $\mu_{n}^{(1)}$ on the interval $\left(0, b_{1}\right)$, for the threshold of low frequencies $\mathcal{T}$ we have $\mathcal{T} \leq b_{1}$. Taking into account this and (4), we deduce from (1) that

$$
\left\|u_{n}^{\varepsilon}\right\|_{\mathcal{H}_{\varepsilon}} \leq c(n) .
$$

Next, with the help of a diagonal process, one can choose a subsequence of $\{\varepsilon\}$ (still denoted by $\{\varepsilon\})$ such that

$$
\left.\begin{array}{l}
\lambda_{n}(\varepsilon) \rightarrow \lambda_{n}^{*} \\
\mathbf{P}_{\varepsilon} u_{n}^{\varepsilon} \rightarrow \tilde{v}_{n}^{(1)}
\end{array}\right\} \quad \text { weakly in } \mathcal{Z}_{0} \text { and strongly in } \mathcal{V}_{0} \text { as } \varepsilon \rightarrow 0
$$

Since $\mathcal{T} \leq b_{1}$, we have

$$
0<\frac{1}{C_{3}^{2}} \leq \lambda_{1}^{*} \leq \ldots \leq \lambda_{n}^{*} \leq \ldots \leq b_{1}
$$

Passing to the limit in (4) as $\varepsilon \rightarrow 0$ and taking into account condition (C4) we obtain $\left(\tilde{v}_{n}^{(1)}, \tilde{v}_{m}^{(1)}\right) \mathcal{v}_{0}=\delta_{n, m} \quad(n, m \in \mathbb{N})$ whence $\tilde{v}_{n}^{(1)} \neq 0$.

Now pass to the limit in the identity

$$
\lambda_{n}(\varepsilon)\left(A_{\varepsilon} u_{n}^{\varepsilon}, \mathbf{S}_{\varepsilon} v\right)_{\mathcal{H}_{\varepsilon}}=\left(u_{n}^{\varepsilon}, \mathbf{S}_{\varepsilon} v\right)_{\mathcal{H}_{\varepsilon}} \quad\left(v \in \mathcal{Z}_{0}\right) .
$$

Due to conditions (C2) and (C4) and the definitions of the operators $A_{\varepsilon}$ and $A_{0}$, the limit of the left-hand side in (12) is equal to $\lambda_{n}^{*}\left(A_{0} \tilde{v}_{n}^{(1)}, v\right)_{\mathcal{H}_{0}}$. Because of condition (C3), the limit of the right hand-side is equal to $\left(\tilde{v}_{n}^{(1)}, v\right)_{\mathcal{H}_{0}}$. Thus, $\lambda_{n}^{*}$ is a characteristic value of the operator $A_{0}$, and it is from the first series $(6) ; \tilde{v}_{n}^{(1)}$ is the corresponding eigenvector.

2. In order to complete the proof, it remains to show that

$$
\lambda_{n}^{*}=\mu_{n}^{(1)} \quad(n \in \mathbb{N}) .
$$

Let $\mu_{k}^{(1)}=\mu_{k+1}^{(1)}=\ldots=\mu_{k+r-1}^{(1)}$ be a characteristic value of the operator $A_{0}$ with multiplicity $r$. Then, let us prove that there exist exactly $r$ characteristic values of the operator $A_{\varepsilon}$ with regard to their multiplicity, which tend to $\mu_{k}^{(1)}$ as $\varepsilon \rightarrow 0$. This will mean that relations (13) are true.

First assume that there exist $q$ characteristic values $\left\{\lambda_{n_{i}}(\varepsilon): i=1, \ldots, q\right\}$ of the operator $A_{\varepsilon}$ which tend to $\mu_{k}^{(1)}$ and $q>r$. By the preceding arguments, we have

$$
\mathbf{P}_{\varepsilon} u_{n_{i}}^{\varepsilon} \rightarrow \tilde{v}_{n_{i}} \quad \text { weakly in } \mathcal{Z}_{0} \text { as } \varepsilon \rightarrow 0 \quad(i=1, \ldots, q)
$$

where $\tilde{v}_{n_{i}}(i=1, \ldots, q)$ are orthonormal in $\mathcal{V}_{0}$ eigenvectors of the operator $A_{0}$. This means that the eigenvalue $\mu_{k}^{(1)}$ has multiplicity $q$. This is a contradiction. 
Now suppose $q<r$. Applying the second part of [53: Lemma 12] to (11) at $n=k+i$, we obtain that there exists a linear combination

$$
U_{\varepsilon}^{(i)}=\sum_{j=1}^{q} \alpha_{i j}(\varepsilon) u_{n_{j}}^{\varepsilon}, \quad 0<c_{1} \leq \sum_{j=1}^{q} \alpha_{i j}^{2}(\varepsilon) \leq c_{2},
$$

of the eigenvectors $u_{n_{1}}^{\varepsilon}, \ldots, u_{n_{q}}^{\varepsilon}$ of the operator $A_{\varepsilon}$ such that

$$
\left\|R_{\varepsilon}^{k+i}-U_{\varepsilon}^{(i)}\right\|_{\mathcal{V}_{\varepsilon}} \leq c_{i} \varepsilon^{\delta_{0}} \quad(i=0, \ldots, r-1) .
$$

Passing to the limit in the last inequalities over a suitable subsequence of $\{\varepsilon\}$ and taking into account condition (C4), we get

$$
v_{k+i}^{(1)}=\sum_{j=1}^{q} \alpha_{i j}^{*} \tilde{v}_{n_{j}}, \quad 0<c_{1} \leq \sum_{j=1}^{q}\left(\alpha_{i j}^{*}\right)^{2} \leq c_{2} \quad(i=0, \ldots, r-1),
$$

but this contradicts the linear independence of the vectors $v_{k}^{(1)}, v_{k+1}^{(1)}, \ldots, v_{k+r-1}^{(1)}(r$ linearly independent vectors are linear combinations of some $q(q<r)$ linearly independent vectors). Since the above reasoning holds for any subsequence of $\{\varepsilon\}$ chosen at the beginning of the proof, we have $\lambda_{n}(\varepsilon) \rightarrow \mu_{n}^{(1)}$ as $\varepsilon \rightarrow 0$. The theorem is proved

\section{Hausdorff convergence of the spectrum}

It turns out that there exist other converging sequences of characteristic values $\lambda_{n(\varepsilon)}(\varepsilon)$ with $n(\varepsilon) \rightarrow+\infty$ as $\varepsilon \rightarrow 0$; the corresponding oscillations are usually called high frequency oscillations.

Theorem 2. The spectrum of the operator $A_{\varepsilon}$ converges to the spectrum of the operator $A_{0}$ in the Hausdorff sense, i.e.

1. For all $\frac{1}{\mu} \in \sigma\left(A_{0}\right)$ there exists $\frac{1}{\lambda(\varepsilon)} \in \sigma\left(A_{\varepsilon}\right)$ such that $\lambda(\varepsilon) \rightarrow \mu$ as $\varepsilon \rightarrow 0$.

2. If $\frac{1}{\lambda(\varepsilon)} \in \sigma\left(A_{\varepsilon}\right)$ and $\lambda(\varepsilon) \rightarrow \Lambda$ as $\varepsilon \rightarrow 0$, then $\frac{1}{\Lambda} \in \sigma\left(A_{0}\right)$.

Proof. If $\frac{1}{\mu} \in \sigma_{d}\left(A_{0}\right)$, then statement 1 follows from condition (C5) and the first part of [53: Lemma 12], which we apply to the corresponding inequality (11). If $\frac{1}{\mu} \in$ $\sigma_{\text {ess }}\left(A_{0}\right)$, then this statement follows from condition (C6) and [53: Lemma 12].

We prove statement 2 by contradiction. Assume $\frac{1}{\Lambda} \notin \sigma\left(A_{0}\right)$. This means also $\frac{1}{\Lambda} \notin \sigma_{e s s}\left(A_{0}\right)$. Because of $(3), \frac{1}{\Lambda} \neq 0$. Consider the sequence $\left\{\left(u^{\varepsilon}, \lambda(\varepsilon), \Lambda\right): \varepsilon>0\right\}$. Due to condition $(\mathrm{C} 2)$ there exists a vector $\mathbf{P}_{\varepsilon} u^{\varepsilon} \in \mathcal{Z}_{0}$ such that $\left\|\mathbf{P}_{\varepsilon} u^{\varepsilon}\right\|_{\mathcal{Z}_{0}} \leq c_{1}(\Lambda)\left\|u^{\varepsilon}\right\|_{\mathcal{H}_{\varepsilon}}$ at $\varepsilon$ small enough. Since $\left\|u^{\varepsilon}\right\|_{\mathcal{V}_{\varepsilon}}=1$ and the sequence $\{\lambda(\varepsilon)\}$ is bounded, it follows from (1) that $\left\|u^{\varepsilon}\right\|_{\mathcal{H}_{\varepsilon}} \leq \lambda(\varepsilon) \leq c$. Therefore, there exists a subsequence $\left\{\varepsilon^{\prime}\right\}$ such that $\mathbf{P}_{\varepsilon^{\prime}} u^{\varepsilon^{\prime}} \rightarrow u^{0}$ weakly in $\mathcal{Z}_{0}$.

Next, similarly as in the first part of Theorem 1 , we conclude that $u^{0} \neq 0$ and $u^{0}$ is an eigenvector, which corresponds to the characteristic value $\Lambda$ of $A_{0}$, i.e. $\frac{1}{\Lambda} \in \sigma\left(A_{0}\right)$. The theorem is proved 


\section{Asymptotic estimates}

In previous two sections we proved convergence theorems, which show that only points of the spectrum of the operator $A_{0}$ are the accumulation points of the spectrum of the operator $A_{\varepsilon}$ as $\varepsilon \rightarrow 0$. The treshold of low frequencies $\left(\mathcal{T}=b_{1}\right)$ indicates where the low and high frequency limits of the spectrum of $A_{\varepsilon}$ are concentrated. In general, the treshold gives us some information about the perturbation of a spectral problem. The treshold is equal to $+\infty$ for majority spectral problems of perturbation theory, for example, for spectral problems with quickly oscillating coefficients, for spectral problems in perforated domains, and we can say, in some sense, that the perturbation for such problems is not very singular.

For spectral problems in thick junctions, the treshold is equal to some positive number for the Neumann condition on the boundaries of the thin domains [30, 31]; it equals $+\infty$ for the Dirichlet condition [38], and it is equal 0 for the Steklov problem [37].

In this section, we prove asymptotic estimates both for the eigenvalues and the eigenvactors of the starting problem and make more precise the asymptotic behavior of the spectrum of $A_{\varepsilon}$ both near points of the discrete spectrum of the operator $A_{0}$ as well as near points of the essential one. As was shown in [34, 38], a new kind of proper oscillations can be presented near the points of the essential spectrum.

Theorem 3. Let $\mu_{n}^{(1)}=\mu_{n+1}^{(1)}=\ldots=\mu_{n+r-1}^{(1)}$ be a characteristic value of the operator $A_{0}$ with multiplicity $r$ and let $v_{n}^{(1)}, \ldots, v_{n+r-1}^{(1)}$ be corresponding eigenvectors satisfying condition (8). Then there exist positive constants $\varepsilon_{0}, C_{i}(n), c_{0}$ and $\left\{\alpha_{i k}(\varepsilon)\right\}$ such that for all $\varepsilon \in\left(0, \varepsilon_{0}\right)$ the inequalities

$$
\left\|R_{\varepsilon}^{(n+i)}-\sum_{k=0}^{r-1} \alpha_{i k}(\varepsilon) u_{n+k}^{\varepsilon}\right\|_{\mathcal{H}_{\varepsilon}} \leq C_{i}(n) \varepsilon^{\delta_{0}} \quad(i=0, \ldots, r-1)
$$

hold where $0<c_{1} \leq \sum_{k=0}^{r-1} \alpha_{i k}^{2}(\varepsilon) \leq c_{2}$ and $R_{\varepsilon}^{(n+i)}=R_{\varepsilon}\left(v_{n+i}^{(1)}\right)$. For any $n \in \mathbb{N}$ and $\varepsilon$ small enough we have

$$
\left|\lambda_{n}(\varepsilon)-\mu_{n}^{(1)}\right| \leq c(n) \varepsilon^{\delta_{0}} .
$$

Proof. Applying to inequality (11) at $n=n+i$ the second part of [53: Lemma 12] with the number

$$
d=\frac{1}{4} \min \left\{\left|\frac{1}{\mu_{n}^{(1)}}-\frac{1}{\mu_{n-1}^{(1)}}\right|,\left|\frac{1}{\mu_{n}^{(1)}}-\frac{1}{\mu_{n+r}^{(1)}}\right|\right\}
$$

and taking into account Theorem 1 , we get

$$
\left\|\frac{R_{\varepsilon}^{(n+i)}}{\left\|R_{\varepsilon}^{(n+i)}\right\|_{\mathcal{H}_{\varepsilon}}}-U_{\varepsilon}^{(i)}\right\|_{\mathcal{H}_{\varepsilon}} \leq 2 d^{-1} c(i) \varepsilon^{\delta_{0}}, \quad\left\|U_{\varepsilon}^{(i)}\right\|_{\mathcal{H}_{\varepsilon}}=1
$$

where $U_{\varepsilon}^{(i)}$ is a linear combination of eigenvalues $u_{\varepsilon}^{n+i}(i=0, \ldots, r-1)$. Now the first statement of the theorem follows from (16) and (10). 
Since the set of eigenvectors $v_{n+i}^{(1)}(i=0,1, \ldots, r-1)$ makes up a finite orthonormal basis in the proper subspace $N\left(\frac{1}{\mu_{n}^{(1)}}, A_{0}\right)$ and due to condition (C5),

$$
\left\|\mu A_{\varepsilon}\left(R_{\varepsilon} v\right)-R_{\varepsilon} v\right\|_{\mathcal{H}_{\varepsilon}} \leq c_{\max }(n) \varepsilon^{\delta_{0}} \quad \forall v \in N\left(\frac{1}{\mu_{n}^{(1)}}, A_{0}\right) \text { with }\|v\|_{\mathcal{V}_{0}}=1
$$

where the constant $c_{\max }(n)>0$ depends neither on $\varepsilon$ nor on $v$.

Let us show that at $\varepsilon$ small enough there are exactly $r$ characteristic values of the operator $A_{\varepsilon}$ on the segment

$$
I(\varepsilon)=\left\{\mu:\left|\mu-\mu_{n}^{(1)}\right| \leq c_{0}(n) \varepsilon^{\delta_{0}}\right\}, \quad \text { where } c_{0}(n)=2 \sqrt{\mu_{n+r}^{(1)}} c_{\max }(n) .
$$

From Theorem 1 it follows that the characteristic values $\lambda_{n+i}(\varepsilon) \quad(i=0, \ldots, r-1)$ may be in this segment. Assume that some characteristic value does not belong to this segment at $\varepsilon$ small enough; without loss of generality it can be assumed that this is $\lambda_{n+r-1}(\varepsilon)$. Then there exists a subsequence $\left\{\varepsilon^{\prime}\right\}$ such that

$$
\mathbf{P}_{\varepsilon^{\prime}}\left(u_{n+r-1}^{\varepsilon^{\prime}}\right) \rightarrow \tilde{v}_{n+r-1}^{(1)} \quad \text { weakly in } \mathcal{Z}_{0} \text { as } \varepsilon^{\prime} \rightarrow 0
$$

where $\tilde{v}_{n+r-1}^{(1)}$ is some eigenvector from the subspace $N\left(\frac{1}{\mu_{n}^{(1)}}, A_{0}\right)$ and $\left\|\tilde{v}_{n+r-1}^{(1)}\right\| \mathcal{V}_{0}=1$. Consider the vector $R_{\varepsilon^{\prime}} \tilde{v}_{n+r-1}^{(1)}$ instead of $v$ in condition (C5). Using the definition of the operator $A_{\varepsilon}$, it follows from (1) that

$$
\begin{aligned}
& \left(\lambda_{n+r-1}\left(\varepsilon^{\prime}\right)-\mu_{n}^{(1)}\right)\left(R_{\varepsilon^{\prime}} \tilde{v}_{n+r-1}^{(1)}, u_{n+r-1}^{\varepsilon^{\prime}}\right)_{\mathcal{V}_{\varepsilon^{\prime}}} \\
& \quad=\left(R_{\varepsilon^{\prime}} \tilde{v}_{n+r-1}^{(1)}-\mu_{n}^{(1)} A_{\varepsilon^{\prime}}\left(R_{\varepsilon^{\prime}} \tilde{v}_{n+r-1}^{(1)}\right), u_{n+r-1}^{\varepsilon^{\prime}}\right)_{\mathcal{H}_{\varepsilon^{\prime}}}
\end{aligned}
$$

Due to (18) and the facts that

$$
\left.\begin{array}{l}
R_{\varepsilon} \tilde{v}_{n+r-1}^{(1)}=\mathbf{S}_{\varepsilon} \tilde{v}_{n+r-1}^{(1)}+\mathcal{O}(\varepsilon) \\
\mathbf{P}_{\varepsilon}\left(\mathbf{S}_{\varepsilon} \tilde{v}_{n+r-1}^{(1)}\right) \rightarrow \tilde{v}_{n+r-1}^{(1)}
\end{array}\right\} \quad \text { in } \mathcal{V}_{0} \text { as } \varepsilon \rightarrow 0
$$

(see condition (C5) and (C2), respectively), we derive from condition (C4) that

$$
\left(R_{\varepsilon^{\prime}} \tilde{v}_{n+r-1}^{(1)}, u_{n+r-1}^{\varepsilon^{\prime}}\right)_{\mathcal{V}_{\varepsilon^{\prime}}}=\left\|\tilde{v}_{n+r-1}^{(1)}\right\|_{\mathcal{V}_{0}}^{2}+\alpha_{\varepsilon^{\prime}}=1+\alpha_{\varepsilon^{\prime}}>\frac{1}{2}
$$

at $\varepsilon$ small enough; here $\alpha_{\varepsilon^{\prime}} \rightarrow 0$ as $\varepsilon^{\prime} \rightarrow 0$. By virtue of (20) and the corresponding inequality for $\tilde{v}_{n+r-1}^{(1)}$ in condition (C5), it follows from (19) that

$$
\begin{aligned}
\left|\lambda_{n+r-1}\left(\varepsilon^{\prime}\right)-\mu_{n}^{(1)}\right| & \leq 2 c_{\max }(n)\left(\varepsilon^{\prime}\right)^{\delta_{0}}\left\|u_{n+r-1}^{\varepsilon^{\prime}}\right\|_{\mathcal{H}_{\varepsilon^{\prime}}} \\
& =2 c_{\max }(n) \sqrt{\lambda_{n+r-1}\left(\varepsilon^{\prime}\right)}\left(\varepsilon^{\prime}\right)^{\delta_{0}} \\
& \leq c_{0}(n)\left(\varepsilon^{\prime}\right)^{\delta_{0}}
\end{aligned}
$$

i.e. $\lambda_{n+r-1}\left(\varepsilon^{\prime}\right) \in I\left(\varepsilon^{\prime}\right)$, but this contradicts our assumption. The theorem is proved 
Remark 3. By condition (C5), $R_{\varepsilon}\left(v_{n+i}^{(1)}\right)=\mathbf{S}_{\varepsilon}\left(v_{n+i}^{(1)}\right)+\mathcal{O}(\varepsilon)$ in $\mathcal{V}_{\varepsilon}$. Now taking into account that the imbedding $\mathcal{H}_{\varepsilon} \subset \mathcal{V}_{\varepsilon}$ is continuous, it follows from (14) (as usually, $\left.\delta_{0} \in(0,1]\right)$ that

$$
\left\|\mathbf{S}_{\varepsilon}\left(v_{n+i}^{(1)}\right)-\sum_{k=0}^{r-1} \alpha_{i k}(\varepsilon) u_{n+k}^{\varepsilon}\right\|_{\mathcal{V}_{\varepsilon}} \leq C_{i}(n) \varepsilon^{\delta_{0}} \quad(i=0, \ldots, r-1) .
$$

The following theorem give us an asymptotic picture near characteristic values of the operator $A_{0}$ from the second series (7).

Theorem 4. Let $\mu_{m}^{(2)}=\mu_{m+1}^{(2)}=\ldots=\mu_{m+r-1}^{(2)}$ be a characteristic value of the operator $A_{0}$ with multiplicity $r$ and let $v_{m}^{(2)}, \ldots, v_{m+r-1}^{(2)}$ be the corresponding eigenvectors, which satisfy (8). Then there exist constants $\varepsilon_{m, k}$ and $c_{3}(m, k)$ such that for any $\varepsilon \in\left(0, \varepsilon_{m, k}\right)$ the interval

$$
I_{m}(\varepsilon)=\left(\mu_{m}^{(2)}-c_{3} \varepsilon^{\delta_{0}}, \mu_{m}^{(2)}+c_{3} \varepsilon^{\delta_{0}}\right)
$$

contains $r$ characteristic values of the operator $A_{\varepsilon}$. Moreover, for the corresponding eigenvectors we have the asymptotic estimates

$$
\left\|\frac{R_{\varepsilon}\left(v_{m+i}^{(2)}\right)}{\left\|R_{\varepsilon}\left(v_{m+i}^{(2)}\right)\right\|_{\mathcal{H}_{\varepsilon}}}-\widetilde{U}_{\varepsilon}^{(i)}\right\|_{\mathcal{H}_{\varepsilon}} \leq c(m) \varepsilon^{\delta_{0}}, \quad\left(\left\|\widetilde{U}_{\varepsilon}^{(i)}\right\|_{\mathcal{H}_{\varepsilon}}=1 ; i=0, \ldots, r-1\right)
$$

where $\widetilde{U}_{\varepsilon}^{(i)}$ is a linear combination of eigenvectors corresponding to all characteristic values of the operator $A_{\varepsilon}$ from the interval $I_{m}(\varepsilon)$.

Proof. The proof is based on arguments of the previous theorems. Namely, we assume that there exist $q$ characteristic values $\lambda_{n_{i}(\varepsilon)}(\varepsilon) \quad(i=1, \ldots, q)$ of the operator $A_{\varepsilon}$, which converge to $\mu_{m}^{(2)}$. Then due to condition $(\mathrm{C} 2)$ we have

$$
\left\|\mathbf{P}_{\varepsilon} u_{n_{i}(\varepsilon)}^{\varepsilon}\right\|_{\mathcal{Z}_{0}} \leq c_{1}\left(\mu_{m}^{(2)}\right)\left\|u_{n_{i}(\varepsilon)}^{\varepsilon}\right\|_{\mathcal{H}_{\varepsilon}} \quad(i=1, \ldots, q) .
$$

In the same way as in the second part of Theorem 1 we prove that $q=r$. To conclude the proof, it remains to repeat the proof of Theorem 3

Remark 4. In fact asymptotic estimate (21) is the estimate of the opening between the subspace $\mathbf{S}_{\varepsilon}\left(N\left(\frac{1}{\mu_{n}^{(1)}}, A_{0}\right)\right)$ and the subspace spanned on eigenvectors of $A_{\varepsilon}$, for which the corresponding eigenvalues tend to $\frac{1}{\mu_{n}^{(1)}}$ as $\varepsilon \rightarrow 0$. A similar estimate we can obtain from (22). If the characteristic value of $A_{0}$ in Theorems 3 or 4 is simple, then (14), (21) or $(22)$ are asymptotic estimates for the eigenvectors of the operator $A_{\varepsilon}$. Usually the norm in $\mathcal{H}_{\varepsilon}$ is the energy norm, and for many problems estimates in the energy norms serve as a criterion that the limiting problem is true.

To obtain asymptotic estimates near the essential spectrum of $A_{0}$, we should use condition (C6), where from we get

$$
\left\|A_{\varepsilon}\left(\frac{w_{\varepsilon}}{\left\|w_{\varepsilon}\right\|_{\mathcal{H}_{\varepsilon}}}\right)-\frac{1}{\mu} \frac{w_{\varepsilon}}{\left\|w_{\varepsilon}\right\|_{\mathcal{H}_{\varepsilon}}}\right\|_{\mathcal{H}_{\varepsilon}} \leq c \varepsilon^{\delta_{1}} .
$$

Applying to this inequality [53: Lemma 12] with the constant $d=c \varepsilon^{\delta_{1} / 2}$, we come to the following theorem. 
Theorem 5. For any $\frac{1}{\mu} \in \sigma_{\text {ess }}\left(A_{0}\right)$ there exists a finite number of eigenvalues of $A_{\varepsilon}$ on the interval

$$
I_{\mu}(\varepsilon)=\left(\frac{1}{\mu}-c \varepsilon^{\delta_{1}}, \frac{1}{\mu}+c \varepsilon^{\delta_{1}}\right) .
$$

Further, there exists a vector $\widetilde{U}_{\varepsilon} \in \mathcal{H}_{\varepsilon}$ such that

$$
\left\|\frac{w_{\varepsilon}}{\left\|w_{\varepsilon}\right\|_{\mathcal{H}_{\varepsilon}}}-\widetilde{U}_{\varepsilon}\right\|_{\mathcal{H}_{\varepsilon}} \leq 2 \varepsilon^{\delta_{1} / 2} \quad \text { and } \quad\left\|\widetilde{U}_{\varepsilon}\right\|_{\mathcal{H}_{\varepsilon}}^{2}=1
$$

and $\widetilde{U}_{\varepsilon}$ is a finite linear combination of eigenvectors $u_{k(\varepsilon)+i}^{\varepsilon}(i=0, \ldots, p(\varepsilon))$ which correspond to all eigenvalues $\lambda_{k(\varepsilon)+i}^{-1}(\varepsilon) \quad(i=0, \ldots, p(\varepsilon))$ of the operator $A_{\varepsilon}$ from the segment $\left[\frac{1}{\mu}-c \varepsilon^{\delta_{1} / 2}, \frac{1}{\mu}+c \varepsilon^{\delta_{1} / 2}\right]$.

\section{Comments}

Here we demonstrate the application of the presented scheme to the problem of the paper [35]. There a mixed boundary value problem for the Laplace operator in a junction $\Omega_{\varepsilon}$ of a domain $\Omega_{0}$ and a large number $N^{2}$ of $\varepsilon$-periodically situated thin cylinders with thickness of order $\varepsilon=O\left(N^{-1}\right)$ was considered.

The operator $\mathbf{P}_{\varepsilon}$ is an extension operator from $H^{1}\left(\Omega_{\varepsilon}\right)$ to $H^{1}\left(\Omega_{0} \cup D_{-}\right)$, where $D_{-}$ is a domain that is filled up by the thin cylinders in the limiting passage as $\varepsilon \rightarrow 0$. This operator is only asymptotically bounded in $\varepsilon$ on the eigenfunctions in the Sobolev space $H^{1}$. It is to be noticed that the construction of the extension operator for junctions of type $3: 2: 1$ is both, the most complex with respect to its construction and the most general of other types of periodic thick junctions.

The operator $\mathbf{S}_{\varepsilon}$ is a restriction operator, which associates with each function $f \in$ $L_{2}\left(\Omega_{0} \cup D_{-}\right)$its restriction to $L_{2}\left(\Omega_{\varepsilon}\right)$.

The operator $R_{\varepsilon}$ is a global asymptotic approximation for the eigenfunctions, which we constructed on the basis of solutions of the limiting spectral problem using the matching principle for the asymptotic expansions. In [37] such approximation was constructed for the essential spectrum of the limiting problem.

\section{References}

[1] Allaire, G., Conca, C. and M. Vanninathan: Spectral asymptotics of the Helmholtz model in fluid-solid structures. Int. J. Numer. Meth. Engng. 46 (1999), 1463 - 1504.

[2] Arrieta, J. M.: Neumann eigenvalues problems on exterior perturbations of the domain. J. Diff. Equ. 118 (1995), 54 - 103.

[3] Babushka, I. and R. Vyborny: Continuous dependence of eigenvalues on the domains. Czech. Math. J. 15 (1965), 169 - 178.

[4] Babych, N. O. and Yu. D. Golovaty: Complete WKB asymptotics of the high frequency vibrations in a stiff problem. Math. Studii. 13 (2000)2, $59-72$. 
[5] Belyaev, A. G.: The asymptotics of solutions of boundary-value problems in periodically perforated domains with small holes (in Russian). Trudy Seminara imeni I.G. Petrovskogo 17 (1994), 153 - 209.

[6] Benkaddour, A. and J. Sanchez-Hubert: Spectral study of a coupled compact-noncompact problem. Model Math. Anal. Number 26 (1992), 659 - 672.

[7] Bourquin, F. and P. G. Ciarlet: Modeling and justification of eigenvalue problems for junctions between elastic structures. J. Funct. Anal. 87 (1989), 392 - 427.

[8] Chechkin, G. A.: Homogenization of boundary value problems with singular perturbation of boundary conditions (in Russian). Mat. Sbornik 184 (1993)6, 99 - 150.

[9] Courant, R. and D. Hilbert: Methods of Mathematical Physics, Vol. 1. New York: Intersci. 1966.

[10] Fleury, F. and E. Sanchez-Palencia: Asymptotic and spectral properties of the acoustic vibrations of body, perforated by narrow channels. Bull. Sci. Math. 110 (1986), $149-176$.

[11] Gadyl'shin, R. R.: On eigenvalues of bodies with thin branches. Part I: Convergence and estimates (in Russian). Mat. zametki 54 (1993)6, $10-21$.

[12] Golovatyi, Yu. D., Nazarov, S. A. and O. A. Oleinik: Asymptotic expansions of eigenvalues and eigenfunctions in problems on oscillations of a medium with concentrated perturbations. Proc. Steklov Math. Inst. 3 (1992), 43 - 63.

[13] Golovaty, Yu. D. and A. S. Lavrenyuk: Asymptotic expansions of local proper vibrations for a plate with density perturbed in neighborhood of one-dimensional manifold. Math. Studii. 13 (2000), 51 - 62.

[14] Hempel, R., Seco, L. and B. Simon: The essential spectrum of Neumann Laplacians on some bounded singular domains. J. Funct. Anal. 102 (1991), 448 - 483.

[15] Jimbo, S.: The singularly perturbed domain and the characterization for the eigenfunctions with Neumann boundary conditions. J. Diff. Equ. 77 (1989), 322 - 350.

[16] Kamotskii, I. V. and S. A. Nazarov: Spectral problems in singularly perturbed domains and self-adjoint extensions of differential operators (in Russian). Trudy Sankt-Petersburg Mat. Obshch. 6 (1998), $151-212$.

[17] Kato, T.: Perturbation Theory for Linear Operators. New York: Springer-Verlag 1966.

[18] Kozlov, V. A., Maz'ya, V. G. and A. B. Movchan: Asymptotic analysis of a mixed boundary value problem in a multi-structure. Asym. Anal. 8 (1994), 105 - 143.

[19] Kozlov, V. A., Maz'ya, V. G. and A. B. Movchan: Asymptotic representation of an elastic field in a multi-structure. Asym. Anal. 11 (1995), 343 - 415.

[20] Leal, C. and E. Sanchez-Palencia: Perturbation of the eigenvalues of a membrane with concentrated mass. Quart. Appl. Math. 47 (1989), 93 - 103.

[21] Lobo, M. and E. Perez: On vibrations of a body with many concentrated masses near the boundary. Math. Models Meth. Appl. Sci. 3 (1993), 249 - 273.

[22] Lobo, M. and E. Perez: Vibrations of a membrane with many concentrated masses near the boundary. Math. Models Meth. Appl. Sci. 5 (1995), 565 - 585.

[23] Lobo, M. and E. Perez: High frequency vibrations in a stiff problem. Math. Models and Methods in Appl. Sci. 7 (1997), 291 - 311.

[24] Maslov, V. P.: Théorie des perturbations et métodes asymptotiques. Paris: Dunod 1972.

[25] Maz'ya, V. G., Nazarov, S. A. and B. A. Plamenewski: Asymptotic expansions of eigenvalues of boundary value problems for the Laplace operator in domains with small holes (in Russian). Izvestijia Acad. Nauk SSSR, ser. Math. 48 (1984), 347 - 371. 
[26] Mel'nyk, T. A.: Averaging of elliptic equations describing the processes in strongly nonuniform thin perforated domains with rapidly varying thickness (in Russian). Dopovidi Akad. Nauk Ukraine. 10 (1991), 15 - 19.

[27] Mel'nyk, T. A. and S. A. Nazarov: Asymptotic structure of spectrum of the Neumann problem in the comb-like domain (in Russian). In: Proc. Conf. "Modern Methods in the Theory of Boundary Value Problems". Voronezh: Math. Institut 1992, pp. $74-75$.

[28] Mel'nyk, T. A. and S. A. Nazarov: The asymptotic structure of the spectrum in the problem of harmonic oscillations of a hub with heavy spokes (in Russian). Dokl. Akad. Nauk Russia. 333 (1993)1, 13 - 15; Engl. transl. in: Russian Acad. Sci. Dokl. Math. 48 (1994), $428-432$.

[29] Mel'nyk, T. A.: Asymptotic expansions of eigenvalues and eigenfunctions for elliptic boundary-value problems with rapidly oscillating coefficients in a perforated cube (in Russian). Trudy Seminara imeni I.G. Petrovskogo 17 (1994), 51 - 88; Engl. transl. in J. Math. Sci. 75 (1995), 1646 - 1671.

[30] Mel'nyk, T. A. and S. A. Nazarov: Asymptotic structure of the spectrum of the Neumann problem in a thin comb-like domain. C.R. Acad. Sci. Paris (Serie 1) 319 (1994), 1343 1348.

[31] Mel'nyk, T. A. and S. A. Nazarov: The asymptotics of the solution of the Neumann spectral problem in a domain of the "dense-comb" type (in Russian). Trudy Seminara imeni I.G. Petrovskogo 19 (1996), 138 - 173; Engl. transl. in: J. Math. Sci. 85 (1997), $2326-2346$.

[32] Mel'nyk, T. A.: Asymptotic analysis of the spectral boundary-value problems in the periodic thick singularly degenerate junctions of the different types. In: Multiple Scale Analyses and Coupled Physical Systems. Proc. Saint-Venant Symp. Paris, August 28 - 29, 1997. Presses des Ponts et Chaussees 1997, pp. 453 - 459.

[33] Mel'nyk, T. A.: Homogenization of the Poisson equation in a thich periodic junction. Z. Anal. Anw. 18 (1999), 953 - 957.

[34] Mel'nyk, T. A.: Decomposing vibrations of thick periodic junctions with concentrated masses. Abstract of the International Workshop "ANASTHEM" (Asymptotic and numerical analysis of structures and of heterogeneous media), June 26 - 30, 2000. Saint Petersburg 2000, pp. $42-45$.

[35] Mel'nyk, T. A.: Asymptotic analysis of a spectral problem in a periodic thick junction of type 3:2:1. Math. Meth. in the Appl. Sci. 23 (2000), 341 - 346.

[36] Mel'nyk, T. A. and S. A. Nazarov: Asymptotic analysis of the Neumann problem in junction of body with thin heavy rods (in Russian). Algebra i Analiz 12 (2000), 188 - 238

[37] Mel'nyk, T. A.: Asymptotic behavior of eigenvalues and eigenfunctions of the Steklov problem in a thick periodic junction. Nonlin. Oscill. 4 (2001)1, $91-105$.

[38] Mel'nyk, T. A.: Vibrations of a thick periodic junction with concentrated masses. Math. Models and Meth. in Appl. Sci. 11 (2001), 1001 - 1029.

[39] Nazarov, S. A. and B. A. Plamenevskii: Asymptotics of the spectrum of the Neumann problem in singularly degenerating thin domains (in Russian). Algebra and Analiz 2 (1990)2, $85-111$.

[40] Nazarov, S. A.: Interaction of concentrated masses in a harmonically oscillating spatial body with Neumann boundary conditions. Model. Math. Anal. Numer. 27 (1993), 777 799. 
[41] Nazarov, S. A. and M. V. Oliushin: On perturbation of eigenvalues of the Neumann problem due to variation of the boundary of a domain (in Russian). Alg. i Anal. 5 (1993), $169-188$.

[42] Oleinik, O. A., Yosifian, G. A. and A. A. Shamaev: Mathematical Problems in Elasticity and Homogenization. Amsterdam: North-Holland 1992.

[43] Ozawa, S.: Spectra of domains with small spherical Neumann boundary. J.Fac. Sci. Univ. Tokyo. 30 (1983), $259-277$.

[44] Rappaz, J., Sanchez-Hubert, J., Sanchez-Palencia, E. and D. Vassiliev: On spectral pollution in the finite element approximation of thin elastic "membrane" shells. Numer. Math. 75 (1997), 473 - 500.

[45] Reed, M. and B. Simon: Methods of Modern Mathematical Physics. Vol. IV: Analysis of Operators. New York et al: Acad. Press 1978.

[46] Rozemblum, G. V., Solomiak, M. Z. and M. A. Shubin: Spectral Theory of Differential Operators (in Russian). Itogi nauki i techniki VINITI. Sovrem. probl. matem. Fundam. napravlenia. 64 (1989), 1 - 242.

[47] Sanchez-Palencia, E.: Non-Homogeneous Media and Vibration Theory. New York: Springer-Verlag 1980.

[48] Sanchez-Palencia, E.: Perturbation of eigenvalues in thermo-elasticity and vibration of systems with concentrated masses. Lect. Notes Phys. 195 (1984), 346 - 368.

[49] Sanchez-Hubert, J. and E. Sanchez-Palencia: Vibration and Coupling of Continuous Systems. Asymptotic Methods. Berlin - Heidelberg: Springer-Verlag 1989.

[50] Simon, B.: Fifty years of eigenvalue perturbation theory. Bulletin Amer. Math. Soc. 24 (1991), $303-319$.

[51] Vanninathan, M.: Homogeneisation des valeurs propres dans les milieux perfores. C.R. Acad. Sci. Paris A 287 (1978), 403 - 406.

[52] Vanninathan, M.: Homogeneisation des problems de valeurs propres dans les milieux perfores. Probleme de Dirichlet. C.R. Acad. Sci. Paris A 287 (1978), 823 - 825.

[53] Vishik, M. I. and L. A. Lyusternik: Regular degeneration and boundary layer for linear differential equations with a parameter (in Russian). Uspekhi Mat. Nauk. 12 (1957)5, 3 $-192$.

[54] Zhikov, V. V., Kozlov, S. M. and O. A. Oleinik: Homogenization of Differential Operators and Integral Functionals. Berlin: Springer-Verlag 1994.

Received 15.02.2001 